\title{
BMJ Open Protocol for a scoping review to understand how interorganisational electronic health records affect hospital physician and pharmacist decisions
}

\author{
Philip Scott, ${ }^{1}$ Haythem Nakkas, ${ }^{1}$ Paul Roderick ${ }^{2}$
}

To cite: Scott P, Nakkas $\mathrm{H}$, Roderick P. Protocol for a scoping review to understand how interorganisational electronic health records affect hospital physician and pharmacist decisions. BMJ Open 2019;9:e023712. doi:10.1136/ bmjopen-2018-023712

- Prepublication history and additional material for this paper are available online. To view please visit the journal (http:// dx.doi.org/10.1136/bmjopen2018-023712).

Received 19 April 2018

Revised 24 October 2018

Accepted 20 November 2018

Check for updates

(C) Author(s) (or their employer(s)) 2019. Re-use permitted under CC BY-NC. No commercial re-use. See rights and permissions. Published by BMJ.

${ }^{1}$ Centre for Healthcare Modelling and Informatics, University of Portsmouth, Portsmouth, UK

${ }^{2}$ Public Health Sciences and Medical Statistics Group, University of Southampton, Southampton, UK

Correspondence to

Dr Philip Scott;

philip.scott@port.ac.uk

\section{ABSTRACT}

Introduction Patient records are often fragmented across organisations and departments in UK health and care services, often due to substandard information technology. However, although government policy in the UK and internationally is strongly pushing 'digital transformation', the evidence for the positive impact of electronic information systems on cost, quality and safety of healthcare is far from clear. In particular, the mechanisms by which information availability is translated into better decision-making are not well understood. We do not know when a full interorganisational record is more useful than a key information summary or an institutional record. In this paper, we describe our scoping review of how interorganisational electronic health records affect decision-making by hospital physicians and pharmacists. Methods and analysis This scoping review will follow the Arksey and 0'Malley (2005) methodology. The review has adopted sociotechnical systems thinking and the notion of distributed cognition as its guiding conceptual models. The UK National Institute for Health and Care Excellence Healthcare Databases Advanced Search will be used, as it incorporates key sources including PubMed, Medline, Embase, HMIC and Health Business Elite. A hand search will be conducted using the reference lists of included studies to identify additional relevant articles. A twopart study selection process will be used: (1) a title and abstract review and (2) full text review. During the first step, two researchers separately will review the citations yielded from the search to determine eligibility based on the defined inclusion and exclusion criteria. Related articles will be included if they are empirical studies that address how interorganisational records affect decisionmaking by hospital physicians and pharmacists.

Ethics and dissemination The results will be disseminated through stakeholder meetings, conference presentations and peer-reviewed publication. The data used are from publicly available secondary sources, so this study does not require ethical review.

\section{INTRODUCTION}

In the current UK National Health Service (NHS) and social care, patient and service user information is fragmented across organisations and departments. Frequently, only the person or their carer knows where the

\section{Strengths and limitations of this study}

- This is a novel scoping review to understand how interorganisational electronic health records affect hospital physician and pharmacist decision-making.

- This review will extend the use of the Distributed Cognition for Teamwork-Concentric Layers framework to secondary research.

- Stakeholders including hospital clinicians, general practitioners, information technology leadership and patient and public representatives will be involved throughout the study.

- The identification and synthesis of data will be limited to peer-reviewed published literature found in the National Institute for Health and Care Excellence Healthcare Databases Advanced Search and snowball references.

- Our defined scope may exclude important aspects of the use of interorganisational electronic health records, both for inpatient and ambulatory care.

various pockets of data about them or their loved ones are hidden away. ${ }^{1}$ This is sometimes due to poor implementation of information technology (IT) ${ }^{2}$ and sometimes due to the implementation of poor IT. ${ }^{34}$ In an era of rising patient and treatment complexity from population ageing, multimorbidity, therapeutic advances and subspecialisation of care, it is in principle reasonable to aim for integrated patient records across health and care to enable more effective and efficient decision-making.

Health and care services are sometimes painted as digital innovation 'laggards' compared with industries like banking, retail and transport. Many UK health services have been digital for years, such as general practice, pathology laboratories and diagnostic imaging. However, it is well known that the level of sustained digital investment in sectors outside health has been massively higher over many decades (at least in the UK). ${ }^{5}$ Also, the culture and structure of the NHS have 
worked against top-down digital transformation (as the failed NHS Connecting for Health programme demonstrated so tragically). ${ }^{26}$

Although government policy in the UK and internationally is strongly pushing 'digital transformation', the overall evidence for the impact of electronic information systems on cost, quality and safety of healthcare remains contested. ${ }^{78}$ Interpretation of the conflicting evidence base is split between aspirational "believers ${ }^{9}$ and more cautious evaluators. ${ }^{510}$

\section{STUDY RATIONALE}

While it seems intuitively obvious that having more information about a patient will improve care, the mechanisms by which information availability is translated into better decision-making are not well understood. ${ }^{11}$ Furthermore, there is the risk of information overload creating a negative outcome. ${ }^{12}$ There are situations where a summary can be more useful than a rich record, ${ }^{13}$ but we do not have a clear synthesis of when or how a full interorganisational record is more useful than a key information summary or an institutional record.

Interorganisational electronic health records (EHRs) are widely implemented in several countries, with the aim to improve the quality of care and reduce costs. However, the literature on their evaluation post implementation is still sparse. ${ }^{14} 15$ This scoping review aims to help understand how interorganisational EHRs can support improvements in direct patient care and how this can inform regional and national information strategies for policy-makers.

The population of interest is hospital physicians and clinical pharmacists, as we are primarily concerned with decisions relating to inpatient diagnosis and treatment.

\section{STUDY OBJECTIVES}

The objective of this scoping review is to identify, categorise, summarise and synthesise knowledge about the mechanisms of action of interorganisational EHRs on decision-making by hospital physicians and pharmacists. Interorganisational EHRs take various forms ${ }^{16}$ : some are static aggregations or repositories of data from multiple institutional records ${ }^{17}$ and others are virtual records dynamically assembled by querying external distributed databases using either proprietary or standards-based semantic interoperability. ${ }^{18} 19$ This review does not distinguish between these types of record, as the focus is on the use of information in decision-making rather than the technical architecture.

\section{METHODS AND ANALYSIS}

\section{Theoretical frameworks}

This scoping review has adopted sociotechnical systems thinking ${ }^{20}$ and in particular the notion of distributed $\operatorname{cognition}^{21}$ as its guiding conceptual models.

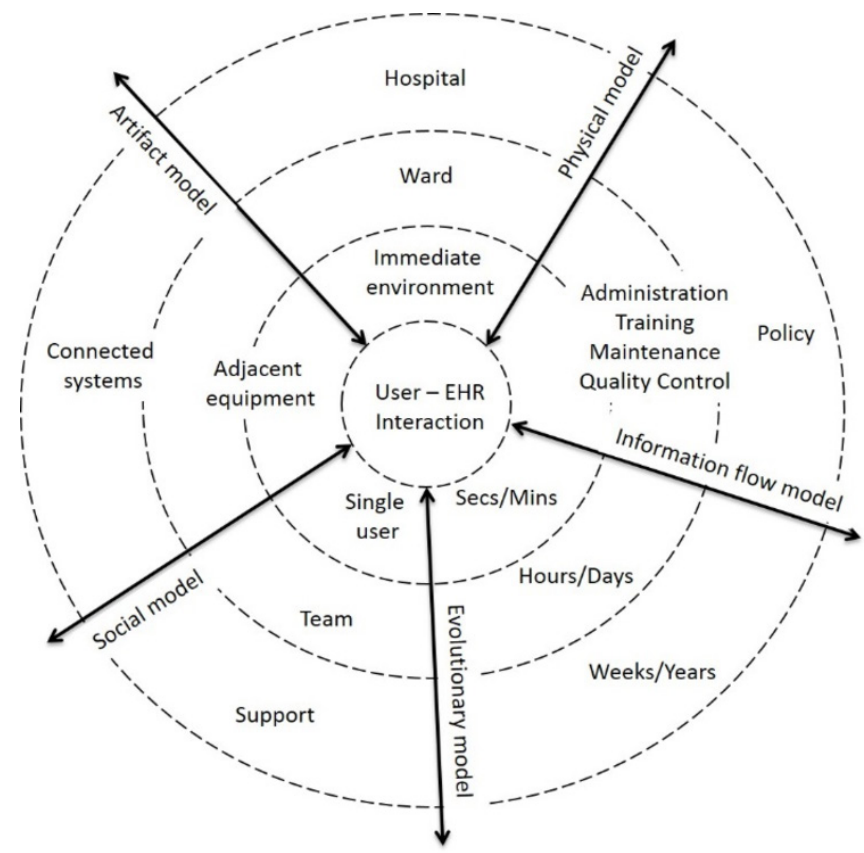

Figure 1 Distributed Cognition for Teamwork-Concentric Layers (adapted from Ref. 26 with permission). EHR, electronic health record.

Sociotechnical systems thinking examines the "dynamic, mutual influences among the social subsystem (people, tasks, relationships), the technical subsystem (technologies, techniques, task performance methods, work settings), and their social and organisational environments'. ${ }^{22}$ It provides the fundamental insights that a technical system inevitably affects and is affected by the interdependent social system within which and on which it operates ${ }^{23}$ and that the sociotechnical system is adaptive and complex (that is, subject to emergent change, not merely complicated). ${ }^{24}$ Distributed cognition has been defined as a paradigm that 'locates thought as an emergent property of people interacting with other actors and the environment rather than a process inherently restricted to individual minds' ${ }^{25}$ In contrast to classical cognitive theory that is constrained to "what goes on in the head', distributed cognition describes what goes on 'in the world' as an interactive cognitive system comprising people, artefacts and environments, and explores 'how information processing is coordinated in sociotechnical systems'. ${ }^{26}$

Specifically, the review will use the framework called Distributed Cognition for Teamwork-Concentric Layers (DiCoT-CL), ${ }^{26}$ shown in figure 1 (based on Ref. 26, adapted with permission). The idea of the concentric circles draws on Grudin's description of technology 'reaching out' through layers of context or abstraction. ${ }^{27}$ This might be interpreted as a 'ripple effect' spreading from the micro to the macro without any fixed boundary. The framework encompasses five submodels: information flow, artefact, physical, social and evolutionary. ${ }^{28}$ Although it was primarily developed to support primary research into teams within a single context, we propose to use the 


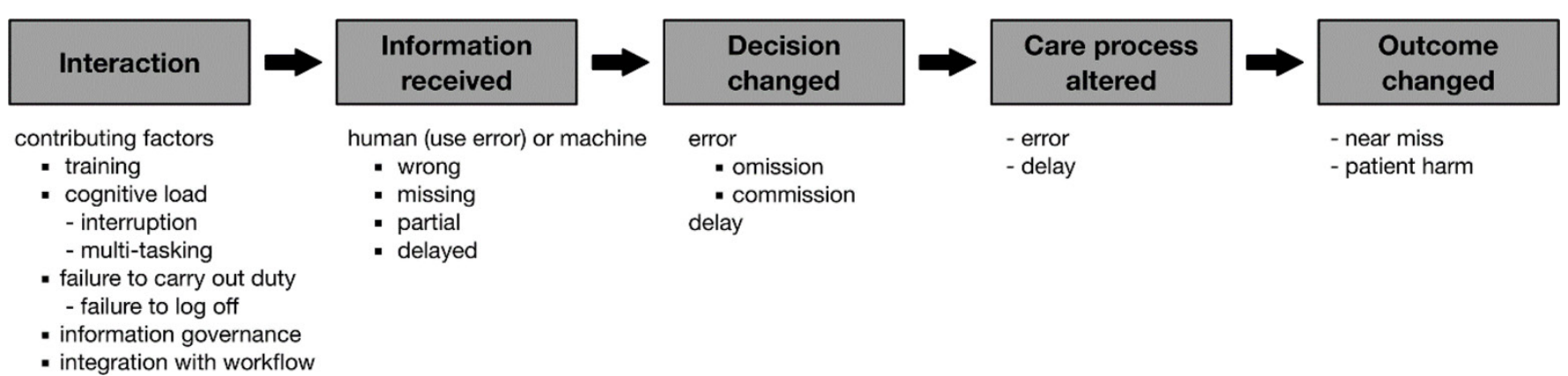

Figure 2 Information value chain (reproduced from ${ }^{29}$ with permission).

framework to guide our analysis and categorisation of the literature across multiple contexts and study types that consider both individual and team decision-making using shared EHRs. We hypothesise that the framework, given its description as leaning towards 'looser use' where the 'boundaries could bend and blur', ${ }^{26}$ will have the flexibility to cope with this extended application for secondary research.

We shall also explore whether it is feasible to combine insights from this framework with any evidence we find that relates to Coiera's notion of the 'information value chain'. The value chain (figure 2, reproduced from ${ }^{29}$ ) might be conceived as a path traversing the DiCoT-CL layers or as an independent axis.

\section{Protocol design}

This study follows the Arksey and O'Malley (2005) scoping review methodology, and various updates. ${ }^{30} 31$ This methodology consists of five stages: (1) identifying the research question(s); (2) identifying potentially relevant studies; (3) selecting eligible studies; (4) charting the data; (5) collating, summarising and reporting the results. This protocol was submitted to PROSPERO, but not accepted for registration as they do not currently take scoping review protocols. The Preferred Reporting Items for Systematic Reviews and Meta-Analysis guidelines ${ }^{32}$ have been followed (as far as relevant for a scoping review) to verify the structure and content of this protocol (the checklist is available as an online supplementary file).

\section{Patient and public involvement}

This review is the first stage of a project which will continue with primary qualitative research into how clinicians actually use a particular interorganisational patient record. We have formed a patient and public involvement (PPI) group to advise the entire project, including the scoping review. The review proposals have been discussed with the project PPI group and with a regional Young Adults PPI group organised by the South Central Research Design Service of the National Institute for Health Research. The PPI discussions confirmed that the proposed scope was important and relevant to patients and that the approach was satisfactory. The project has a steering group with representation from hospital clinicians, general practitioners, the NHS funding body and the IT leadership.
Stage 1: identifying the research questions Arksey and $\mathrm{O}^{\prime} \mathrm{Malley}{ }^{33}$ propose that an iterative process is required in order to formulate the research question(s) and that this will help the researchers to familiarise themselves with the literature. Our research questions were developed and refined through an iterative process and consultations held by the research team. This review will be guided by the main broad research question: (RQ1) 'How do inter-organisational electronic health and care records affect decision-making by hospital physicians and pharmacists?' This question was selected based on the scope of our primary research that will follow this review. We are not presuming that all effects will be positive or making that an inclusion criterion.

Furthermore, two secondary research questions will be used to guide this review, with the same implicit scope: (RQ2) 'When are rich EHRs more useful than summary records?' and (RQ3) 'What specific pathways or protocols demonstrate cost reduction or quality improvement from interorganisational EHRs?'

\section{Stage 2: identifying relevant studies—search terms and inclusion/exclusion criteria}

Based on preliminary searches, the research team will identify and refine eligibility criteria and the formulation of the search strategy and search terms. The currently proposed initial search strategy is shown in online supplementary appendix 1 . The search has been limited to studies published since 2008, but we anticipate locating older or seminal papers about interorganisational EHRs through snowball referencing. This search strategy will be iterated and refined as required to ensure the inclusion of all the relevant studies from the literature. Search results will be downloaded and imported into Microsoft Excel for further analysis.

We have selected the UK National Institute for Health and Care Excellence Healthcare Databases Advanced Search (HDAS), as it incorporates key sources including PubMed, Medline, Embase, HMIC and Health Business Elite. Furthermore, hand searches will be conducted using the reference lists of the included studies in order to identify additional relevant articles that may not be directly indexed in HDAS sources.

Articles will be included if they are empirical studies that address how interorganisational EHRs or health 
Table 1 Population, intervention, comparator, outcomes

\begin{tabular}{|c|c|}
\hline Population & Hospital physicians and pharmacists \\
\hline Intervention & Interorganisational electronic health records \\
\hline Comparator & $\begin{array}{l}\text { Usual practice without interorganisational } \\
\text { electronic health records }\end{array}$ \\
\hline Outcomes & $\begin{array}{l}\text { Any outcome relating to changed decisions } \\
\text { or decision-making process in diagnosis and } \\
\text { treatment }\end{array}$ \\
\hline
\end{tabular}

information exchange are used in decision-making by hospital physicians or pharmacists. Studies will be excluded if they are discussing solely the technical aspect of designing EHRs, health information exchange or the clinical decision support systems embedded in EHRs. Studies will also be excluded if they address EHRs or clinical decision support systems within a single organisation. The inclusion criteria defined by population, intervention, comparator and outcomes is shown in table 1.

\section{Stage 3: study selection}

A two-part study selection process will be used: (1) a title and abstract review and (2) full-text review. Inter-rater reliability will be calculated for both stages using Cohen's kappa to iteratively calibrate and refine the process. In the first step, two reviewers will separately review the citations yielded from the search to determine the eligibility based on the defined inclusion and exclusion criteria. To confirm their robustness, the inclusion and exclusion criteria will be tested on a sample of abstracts before conducting the actual search to help capture any studies that may be relevant to interorganisational EHRs. All the articles which are considered relevant by either reviewer will be included in the full-text evaluation.

In the second step, two reviewers will independently evaluate the full-text articles to decide if they meet the inclusion/exclusion criteria. In case of any disagreement about inclusion, full-text articles will be reviewed again by both reviewers and if an agreement cannot be reached, this will be resolved by a consolidation with an independent third reviewer. ${ }^{34}$

\section{Stage 4: charting the data}

The fourth stage of Arksey and O'Malley ${ }^{33}$ scoping review methodology is the charting of the data of the selected articles. A data extraction form will be developed by the reviewers to help in deciding the relevance of the study. During this stage, key information about the selected articles will be collected (eg, author(s), year of publication, country, objectives of the study, methods, findings). The data extraction form will be reviewed by the research team and our stakeholders (steering group and PPI representatives). In order to ensure the validity of the data extraction form, it will be piloted by both reviewers before conducting the actual searches. After the actual searches have been executed, the data extraction will be subjected to a test by both reviewers separately extracting the data from a sample of the included articles. The sample size will be calculated once the total number included is known. ${ }^{30}$ Subject to the outcome of this test and the volume of included papers, the team will determine whether complete independent extraction is necessary or if it can be performed separately.

\section{Stage 5: collating, summarising and reporting the results}

As a scoping review, the purpose of this study is to aggregate the findings and present an overview of the research rather than to evaluate the quality of the individual studies. Our overall assessment of the strength of the evidence will therefore be narrative rather than quantitative. We will use the DiCoT-CL framework and the information value chain concept to help to categorise and synthesise the literature. We will also report our experience with the scoping review methodology and any suggestions for improvement that we might develop.

\section{Limitations}

We recognise that our defined scope, decision-making by hospital physicians or pharmacists, may exclude other important aspects of the use of interorganisational EHRs or health information exchange, both for inpatient and ambulatory care. However, this definition is in line with the nature of our planned primary research and therefore sufficient for the current study. We hope to extend this review with further work with a broader scope in due course.

\section{DISCUSSION}

An evidence-based approach to 'digital health' is still not the norm. ${ }^{5}$ This scoping review aims to contribute to the health informatics evidence base by consolidating knowledge about the impacts of wider and richer information sharing on diagnosis and treatment of hospital inpatients, using the theoretical lenses of distributed cognition and the information value chain. The review will inform our subsequent primary research and contribute useful insights for the design and implementation of future generations of health record.

Acknowledgements The authors gratefully acknowledge the helpful comments of their PPI advisers and peer reviewers.

Contributors PS conceived the study, outlined the protocol and is guarantor of the review. PS and HN jointly developed the research questions and drafted the paper. $\mathrm{PR}$ revised the paper. All authors further revised the paper and approved the final text.

Funding This work is supported by a grant from the NHS Hampshire \& Isle of Wight Sustainability and Transformation Partnership. The funders participated in defining the scope of the review but have not been involved in the detailed protocol development presented in this paper.

Competing interests None declared.

Patient consent Not required.

Provenance and peer review Not commissioned; externally peer reviewed.

Open access This is an open access article distributed in accordance with the Creative Commons Attribution Non Commercial (CC BY-NC 4.0) license, which permits others to distribute, remix, adapt, build upon this work non-commercially, and license their derivative works on different terms, provided the original work is 
properly cited, appropriate credit is given, any changes made indicated, and the use is non-commercial. See: http://creativecommons.org/licenses/by-nc/4.0/.

\section{REFERENCES}

1. The Richmond Group of Charities. My data, my care. How better use of data improves health and wellbeing. 2017. https://richmondgrou pofcharities.org.uk/sites/default/files/Ir_5233_richmond_group_my_ data my care report.pdf (cited 17 Apr 2018).

2. Wachter R. Making IT work: harnessing the power of health information technology to improve care in England. 2016. https:// www.gov.uk/government/publications/using-information-technologyto-improve-the-nhs (cited 18 Apr 2018).

3. Heponiemi T, Hyppönen H, Vehko T, et al. Finnish physicians' stress related to information systems keeps increasing: a longitudinal threewave survey study. BMC Med Inform Decis Mak 2017;17:147.

4. Schiff GD, Amato MG, Equale T, et al. Computerised physician order entry-related medication errors: analysis of reported errors and vulnerability testing of current systems. BMJ Qual Saf 2015;24:264-71.

5. Scott P. Exploiting the information revolution: call for independent evaluation of the latest English national experiment. J Innov Health Inform 2015;22:244-9.

6. Sheikh A, Cornford T, Barber N, et al. Implementation and adoption of nationwide electronic health records in secondary care in England: final qualitative results from prospective national evaluation in "early adopter" hospitals. BMJ 2011;343:d6054.

7. Black AD, Car J, Pagliari C, et al. The impact of eHealth on the quality and safety of health care: a systematic overview. PLoS Med 2011;8:e1000387.

8. Campanella P, Lovato E, Marone C, et al. The impact of electronic health records on healthcare quality: a systematic review and metaanalysis. Eur J Public Health 2016;26:60-4.

9. Hillestad R, Bigelow J, Bower A, et al. Can electronic medical record systems transform health care? Potential health benefits, savings, and costs. Health Aff 2005;24:1103-17.

10. Fillmore CL, Bray BE, Kawamoto K. Systematic review of clinical decision support interventions with potential for inpatient cost reduction. BMC Med Inform Decis Mak 2013;13:135

11. Roshanov PS, Misra S, Gerstein HC, et al. Computerized clinical decision support systems for chronic disease management: a decision-maker-researcher partnership systematic review. Implement Sci 2011;6:92.

12. Singh $\mathrm{H}$, Spitzmueller $\mathrm{C}$, Petersen $\mathrm{NJ}$, et al. Information overload and missed test results in electronic health record-based settings. JAMA Intern Med 2013;173:702-4.

13. Whiting-O'Keefe QE, Simborg DW, Epstein WV, et al. A computerized summary medical record system can provide more information than the standard medical record. JAMA 1985;254:1185-92.

14. Akhlaq A, Sheikh A, Pagliari C. Health information exchange as a complex and adaptive construct: scoping review. J Innov Health Inform 2017;23:633-83.

15. Hripcsak G, Kaushal R, Johnson KB, et al. The United Hospital Fund meeting on evaluating health information exchange. J Biomed Inform 2007;40:S3-S10.
16. Häyrinen K, Saranto K, Nykänen P. Definition, structure, content, use and impacts of electronic health records: a review of the research literature. Int J Med Inform 2008;77:291-304.

17. Sanderson $\mathrm{H}$, Adams $\mathrm{T}$, Budden $\mathrm{M}$, et al. Lessons from the central Hampshire electronic health record pilot project: evaluation of the electronic health record for supporting patient care and secondary analysis. BMJ 2004;328:875-8.

18. Wu CH, Chiu RK, Yeh HM, et al. Implementation of a cloud-based electronic medical record exchange system in compliance with the integrating healthcare enterprise's cross-enterprise document sharing integration profile. Int J Med Inform 2017;107:30-9.

19. HL7 International. FHIR Overview - Architects. 2018 https://www.hl7. org/fhir/overview-arch.html (cited Oct 14 2018)

20. Davis MC, Challenger R, Jayewardene DN, et al. Advancing socio-technical systems thinking: a call for bravery. Appl Ergon 2014;45:171-80.

21. Hazlehurst B, Gorman PN, McMullen CK. Distributed cognition: an alternative model of cognition for medical informatics. Int $\mathrm{J}$ Med Inform 2008;77:226-34.

22. Harrison Ml, Koppel R, Bar-Lev S. Unintended consequences of information technologies in health care--an interactive sociotechnical analysis. J Am Med Inform Assoc 2007;14:542-9.

23. Scott PJ, Briggs JS. STAT-HI: a socio-technical assessment tool for health informatics implementations. Open Med Inform $J$ 2010;4:214-20.

24. Sittig DF, Singh $\mathrm{H}$. A new sociotechnical model for studying health information technology in complex adaptive healthcare systems. Qual Saf Health Care 2010;19(Suppl 3):i68-i74.

25. Lippa KD, Feufel MA, Robinson FE, et al. Navigating the decision space: shared medical decision making as distributed cognition. Qual Health Res 2017;27:1035-48.

26. Furniss D, Masci P, Curzon P, et al. Exploring medical device design and use through layers of distributed cognition: how a glucometer is coupled with its context. J Biomed Inform 2015;53:330-41.

27. Grudin J. The computer reaches out: the historical continuity of interface design. SIGCHI Conference on Human Factors in Computing Systems, Seattle, Washington, USA, 1990.

28. Craven CK, Doebbeling B, Furniss D, et al. Evidence-based health informatics frameworks for applied use. Stud Health Technol Inform 2016;222:77-89.

29. Kim MO, Coiera E, Magrabi F. Problems with health information technology and their effects on care delivery and patient outcomes: a systematic review. J Am Med Inform Assoc 2017;24:246-50.

30. Daudt HM, van Mossel C, Scott SJ. Enhancing the scoping study methodology: a large, inter-professional team's experience with Arksey and O'Malley's framework. BMC Med Res Methodol 2013;13:48

31. Levac D, Colquhoun H, O'Brien KK. Scoping studies: advancing the methodology. Implement Sci 2010;5:69.

32. Shamseer L, Moher D, Clarke M, et al. Preferred reporting items for systematic review and meta-analysis protocols (PRISMA-P) 2015: elaboration and explanation. BMJ 2015;350:g7647.

33. Arksey H, O'Malley L. Scoping studies: towards a methodological framework. Int J Soc Res Methodol 2005;8:19-32.

34. Schlosser RW. Appraising the quality of systematic reviews. Focus 2007;17:1-8. 